\title{
DIABETES MELLITUS: A NECESSIDADE DE UM DISPOSITIVO DE ADMINISTRACÃO DE INSULINA PARA CRIANCAS E ADOLESCENTES
}

\section{DIABETES MELLITUS: A NEED FOR AN INSULIN DELIVERY DEVICE FOR TEENS}

Lucas Renato Tescke ${ }^{1}$ João Manuel R. S. Tavares ${ }^{2}$ Isa C. T. Santos ${ }^{3}$ 


\section{Resumo}

O diabetes mellitus é uma doença crônica em rápida expansão pelo mundo, afetando pessoas de qualquer idade ou sexo. É uma doença que atinge o pâncreas prejudicando a produção de insulina, e em alguns casos o pâncreas para de produzir este hormônio. Apesar de não haver cura, o tratamento da diabetes consiste em injeções diárias de insulina; assim, as corretas administrações de insulina são indispensáveis para manter o controle glicêmico e prevenir futuras complicações. A identificação da oportunidade do desenvolvimento deste projeto é apresentada pela inexistência de um dispositivo de administração de insulina específico para crianças. Este estudo visou a identificação das necessidades para o desenvolvimento de um novo dispositivo de administração de insulina para crianças diabéticas que permitisse aumentar a qualidade de vida das mesmas. Para tal, foi estudado o histórico da doença, identificados dispositivos existentes, levantadas novas tecnologias apara o tratamento da doença e identificadas as necessidades do utilizador.

Palavras-Chave: Diabetes mellitus. Desenvolvimento de dispositivos. Análise das necessidades.

\footnotetext{
${ }^{1}$ Lucas Renato Tescke Instituto Federal Farroupilha lucastescke@hotmail.com

2 João Manuel R. S. Tavares

Instituto de Ciência e Inovação em Engenharia Mecânica e Engenharia Industrial, Faculdade de Engenharia, Universidade do Porto tavares@fe.up.pt
}

\section{Abstract}

Diabetes mellitus is a chronic disease in rapid expansion throughout the world, affecting people of any age or gender. This disease affects the pancreas influencing insulin production, and, in some cases, the pancreas stops producing that hormone. Even though there is no cure, the treatment of diabetes consists of daily injections of insulin, which are necessary to control the glycemic levels and prevent future complications. The identification of an opportunity for the development of this project comes from the inexistence of a specific device for administering insulin on children. This study aimed to identify the needs to development a new insulin delivery device for diabetic children allowing to increase the quality life of them. For such, it was studied the history of the disease, the devices available on the market, new technologies for the treatment of this disease and the necessities of the user.

Keywords: Diabetes mellitus. Devices development. Needs assessment.

ISSN: $1808-3129$

\author{
${ }^{3}$ Isa C. T. Santos \\ Instituto de Ciência e Inovação em Engenharia Mecânica \\ e Engenharia Industrial, Faculdade de Engenharia, Uni- \\ versidade do Porto \\ isa.santos@fe.up.pt
}




\section{INTRODUÇÃO}

O aumento da incidência e prevalência do diabetes mellitus apresenta características de pandemia [1]. O diabetes tipo 1 é desenvolvida em crianças e adolescentes e sua causa está ligada a fatores ambientais [1]. As preocupações são mais evidentes quando diagnosticada o diabetes mellitus tipo 1, pois, na maior parte dos casos, atinge crianças e adolescentes [2]. É neste tipo de diabetes que ocorrem as maiores complicações crónicas, necessitando de cuidado e tratamento rigoroso, o qual deve monitorar e administrar o nível de glicose diariamente [3].

O monitoramento e administração de insulina são realizados através de dispositivos médicos específicos para esta doença, sendo que o utilizador constantemente busca dispositivos que ajudem a melhorar sua qualidade de vida e, também, evitar preconceitos associados à doença. Assim, estes dispositivos são indispensáveis no controle da doença, e fatores como ergonomia, design e praticidade são de fundamental importância à adaptação do utilizador com o dispositivo e o tratamento.

Desenvolver dispositivos médicos requer intenso estudo junto ao utilizador, visando determinar os aspectos específicos do público-alvo. A motivação para o desenvolvimento deste projeto deveu-se a inexistência de dispositivos específicos para o controlo da doença em crianças e adolescentes. Além disso, médicos e agentes de saúde afirmam frequentemente ter grande preocupação na educação à independência do doente tendo êxito no autocontrolo da doença [2].

Com base no estudo apresentado a seguir, viu-se a possibilidade em desenvolver um dispositivo de administração de insulina compatível com as especificidades das crianças, que promova a independência no autocontrole da doença e o aumento da qualidade de vida. O estudo dividiu-se na identificação do histórico da doença e dos dispositivos mais utilizados no tratamento, posteriormente iniciou-se a fase de pesquisa com o utilizador para levantamento e análise das necessidades e, por fim, foram identificados os requisitos para o desenvolvimento de um novo dispositivo especialmente dedicado para crianças. 


\section{DESENVOLVIMENTO}

Hoje em dia, o paciente diabético, tanto criança quanto adulto, recebe o mesmo tipo de dispositivo para realizar a administração de insulina. Os problemas percebidos quando estes dispositivos são utilizados por crianças vão além da estética, a maior preocupação é quanto a ergonomia e funcionalidade, devido a discrepância entre as necessidades dos dois públicos. Para mais, o tratamento é bastante diferenciado entre os diabéticos do tipo 1 e 2, exigindo um maior número de picadas (administrações de insulina) durante o dia nas crianças, influenciando ainda mais na relação objeto e utilizador.

Ergonomia e funcionalidade interferem na qualidade de vida do utilizador e ainda mais no controle da doença. O desenvolvimento dos dispositivos para administração é voltado ao público adulto devido a maior incidência da doença nessa faixa etária, equivalendo a 90\% dos casos de diabetes mellitus tipo 2 [4], restringindo a criação de dispositivos adequados ao público infantil e jovem, que apesar do menor número (10\%) são os que necessitam de dispositivos específicos para seu tratamento.

O principal objetivo deste projeto foi a identificação da necessidade de desenvolvimento de um dispositivo para administração de insulina para crianças com diabetes tipo 1.

\section{Insulinoterapia}

Há mais de 90 anos desde a primeira injeção de insulina em um jovem, tanto a descoberta como o início da terapêutica do diabetes foram um marco na historia da medicina e do diabético [5]. A partir disso, o diabetes que era considerada uma doença fatal passou a ser uma doença crônica de evolução prolongada, devido suas complicações a curto, médio e longo prazo. A descoberta da insulina proporcionou grandes progressos na terapêutica dos diabéticos insulinodependentes, "melhoria da sua qualidade de vida, no desenvolvimento normal das crianças diabéticas" e "na redução de complicações agudas" [5].

A primeira insulina utilizada no tratamento do diabetes foi a insulina regular, porém era necessário realizar diversas aplicações durante o dia. $O$ número de picadas diminui após surgirem as insulinas de ações intermediárias ou de ações mais prolongadas, reduzindo para uma ou duas administrações diariamente [6].

Atualmente, recomenda-se administrar insulina por injeção subcutânea, entre a pele e o músculo [7]. Quando administrada no subcutâneo sua absorção é gradativa e eficaz, quando aplicada em outro tecido, que não o subcutâneo, é alterado o tempo de absorção da insulina e prejudica o controle glicêmico do diabético [4].

\section{Dispositivos mais utilizados e novas tecnologias}

Dentre os dispositivos existentes no mercado, as seringas de insulina são dispositivos bastante simples e de baixo custo. Sua principal desvantagem é a indiscrição durante a administração e a imprecisão das doses. Outro dispositivo são as canetas 
de insulina, são bastante práticas e discretas, além de ser o meio mais utilizado pelos diabéticos para administração de insulina hoje em dia. As bombas de infusão são dispositivos caros e exigem algum treinamento do diabético para entender seu funcionamento, sendo a forma mais prática para administração de insulina, além de reduzir o número de picadas, pois a cânula só é substituída entre 3 e 5 dias, esse método é muito preciso nas doses, ideal para diabéticos de tipo 1.

Para mais, novas formas de administração de insulina estão em desenvolvimento, como via oral, intranasal e como supositório. Entretanto, são pesquisas recentes que não comprovam seu real benefício em longo prazo, fator importantíssimo para pesquisas na área médica.

\section{Identificação e análise das necessidades}

Posteriormente ao entendimento da condição médica, o estudo foi conduzido à investigação das necessidades dos utilizadores, denominada como "voz do consumidor", 3 apresentada por Santos et al. 2012 [8], Figura 1. Através desta análise é possível identificar algumas necessidades dos utilizadores e como o problema do desenvolvimento de um novo dispositivo médico pode ser tratado.

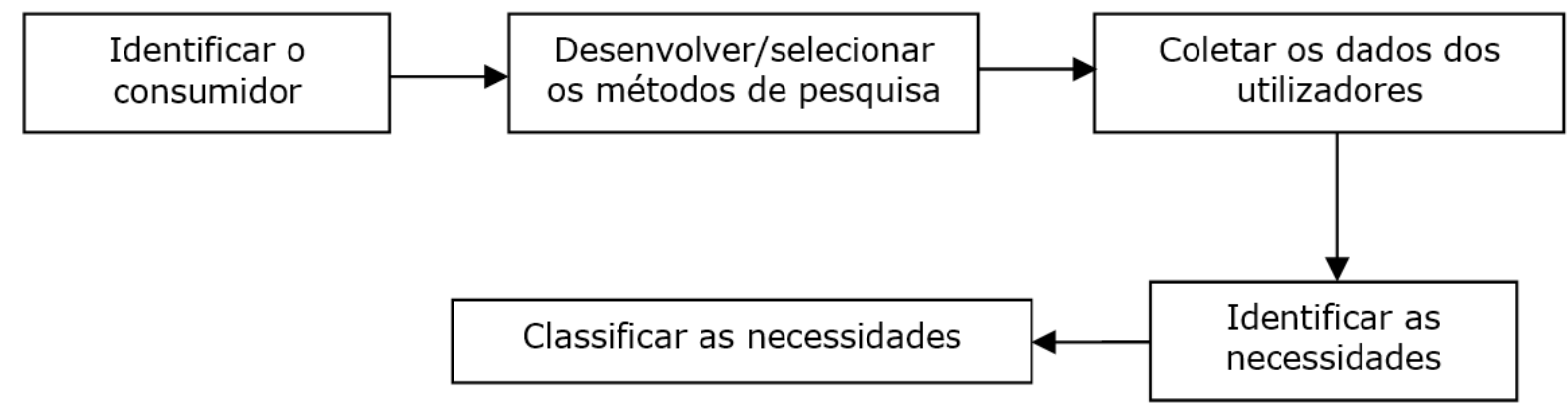

Figura 1: Metodologia para realizar a pesquisa "voz do consumidor" [8]

Para identificar as necessidades dos utilizadores, foram contatadas diversas Associações de Diabéticos, em Portugal, onde fosse possível realizar uma pesquisa de campo e outra a nível virtual (online). A pesquisa participativa envolveu a Associação de Diabéticos do Minho, onde foi disponibilizado o acompanhamento de crianças em uma visita a Aveiro, durante um dia, conseguido uma amostra de 12 inquiridos. Nesta pesquisa houve mais-valia devido ao diálogo realizado com os inquiridos durante o processo de administração de insulina, obtendo dados qualitativos. Os dados recolhidos abordavam questões referentes ao dia-a-dia entre criança e a doença, sua opinião sobre os produtos utilizados na administração de insulina e como desejaria que fosse este dispositivo. Porém, a fim de obter maior número de opiniões realizou-se uma recolha de dados quantitativos através de um questionário online. Este questionário foi realizado em parceria com a Associação de Jovens Diabéticos de Portugal, devido ao grande número de participantes nesta instituição, onde possibilitou a recolha de 60 respostas. Optou-se por não recorrer a Hospitais devido ao 
nível burocrático e demora ao acesso à informação, sendo que em alguns casos era necessária aprovação da comissão de ética, envolvendo muito tempo para tal tarefa.

Os dados foram obtidos por meio de um questionário com questões fechadas e abertas, analisado e aprovado previamente pelas duas Associações.

\section{Voz do consumidor}

Ao todo foram recolhidas 72 respostas, a Tabela 1 descreve a amostra usada.

\begin{tabular}{|c|c|c|c|c|}
\hline & \multicolumn{2}{|c}{ Feminino } & \multicolumn{2}{c|}{ Masculino } \\
\hline $0-14$ anos & 13 & $18 \%$ & 21 & $29 \%$ \\
\hline $15-20$ anos & 8 & $11 \%$ & 2 & $3 \%$ \\
\hline$>21$ anos & 9 & $13 \%$ & 19 & $26 \%$ \\
\hline Total & $\mathbf{3 0}$ & $\mathbf{4 2} \%$ & $\mathbf{4 2}$ & $\mathbf{5 8} \%$ \\
\hline
\end{tabular}

Tabela 1: Gênero e idade dos diabéticos inquiridos

Grande parte dos diabéticos descobriu inquiridos a doença há mais de três anos (74\%), o restante está dividido entre um e dois anos após a descoberta. Os tipos de insulinas mais utilizados para administração por estes diabéticos são insulina lenta (79\%) e insulina rápida (71\%). Geralmente estes dois tipos de insulina são utilizados de forma complementar no tratamento da doença, ou seja, quando utilizado a insulina de ação lenta faz-se necessário o uso de insulina de ação rápida para regularizar os níveis de glicemia ao longo do dia, administrada principalmente antes das refeições (75\%). Consequentemente, com estes tipos de insulina aumenta o número de picadas, verifica-se que cerca de $90 \%$ dos diabéticos administram a insulina quatro ou mais vezes durante o dia. Na maioria das vezes, é o próprio diabético o responsável pela administração (75\%).

\section{Dispositivos de administração de insulina}

Os dispositivos utilizados na administração de insulina são: caneta de insulina (56\%), caneta de insulina descartável (28\%), bomba de infusão contínua (13\%) e somente $4 \%$ utiliza seringa de insulina. A maioria dos diabéticos utiliza apenas um dispositivo para realizar as administrações (74\%), os outros $26 \%$ combinam dois dispositivos, sendo: $50 \%$ canetas de insulina reutilizáveis, $47 \%$ caneta de insulina descartável e $3 \%$ seringa de insulina. A escolha do dispositivo é definida na maioria dos casos pelo médico (79\%) e não pelo próprio doente. O fator motivador da escolha do dispositivo é dividido da maneira destacada na Figura 2. 


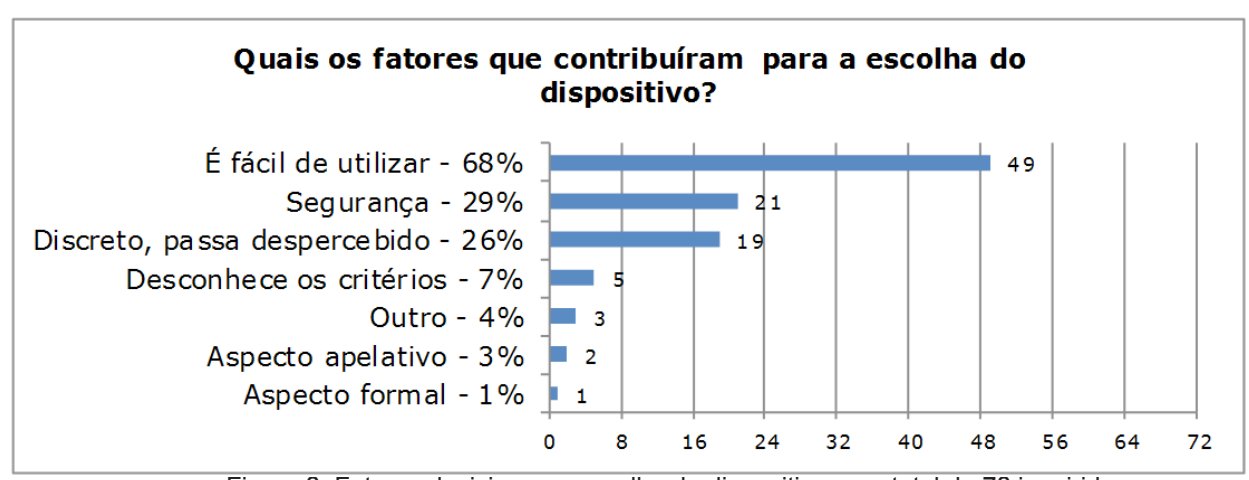

Figura 2: Fatores decisivos na escolha do dispositivo num total de 72 inquiridos

Apesar do nível de satisfação, Figura 3, em relação ao dispositivo utilizado ser bom, percebem-se muitas queixas dos utilizadores quanto ao número de picadas diárias, apesar de já estarem acostumados, o constante monitoramento da glicose e o tamanho dos dispositivos. Foi questionado também como o diabético sente-se durante a administração, muitos deles referem-se a não se sentirem incomodados ao realizar a administração de insulina em frente a outras pessoas. Porém, gostariam que o dispositivo fosse mais discreto para não transmitir a sensação de "doença".

Figura 3: Nível de satisfação do dispositivo utilizado para um total de 72 respostas

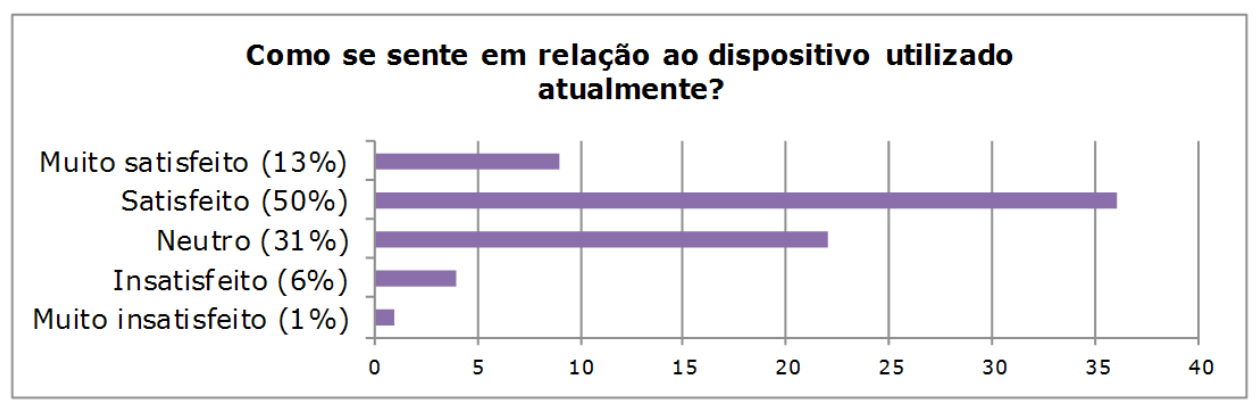

Entretanto, a maior dificuldade dos diabéticos é no fator de insegurança durante a administração (44\%), os doentes não se sentem seguros diante da tarefa diária para controlar o nível glicêmico através das injeções de insulina, devido o cálculo realizado para saber a dosagem correta. Por outro lado, cerca de $28 \%$ apresentam medo das diversas picadas que devem superar diariamente.

\section{Análise dos produtos mais utilizados pelos diabéticos}

Através da análise dos produtos similares realizou-se uma tabela comparativa entre os produtos existentes no mercado, as suas principais características e os requisitos definidos pelo utilizador. Esta análise ajuda a perceber os pontos positivos e negativos de cada dispositivo, além de compreender quais características fazem com que o produto alcance o sucesso. Para isso, foi feita uma escala de pontuação entre, positivo "1", neutro "0" e negativo "-1" para cada característica do produto comparada com os pré-requisitos, Tabela 2 e Tabela 3, os critérios foram definidos pelas principais características dos dispositivos. 


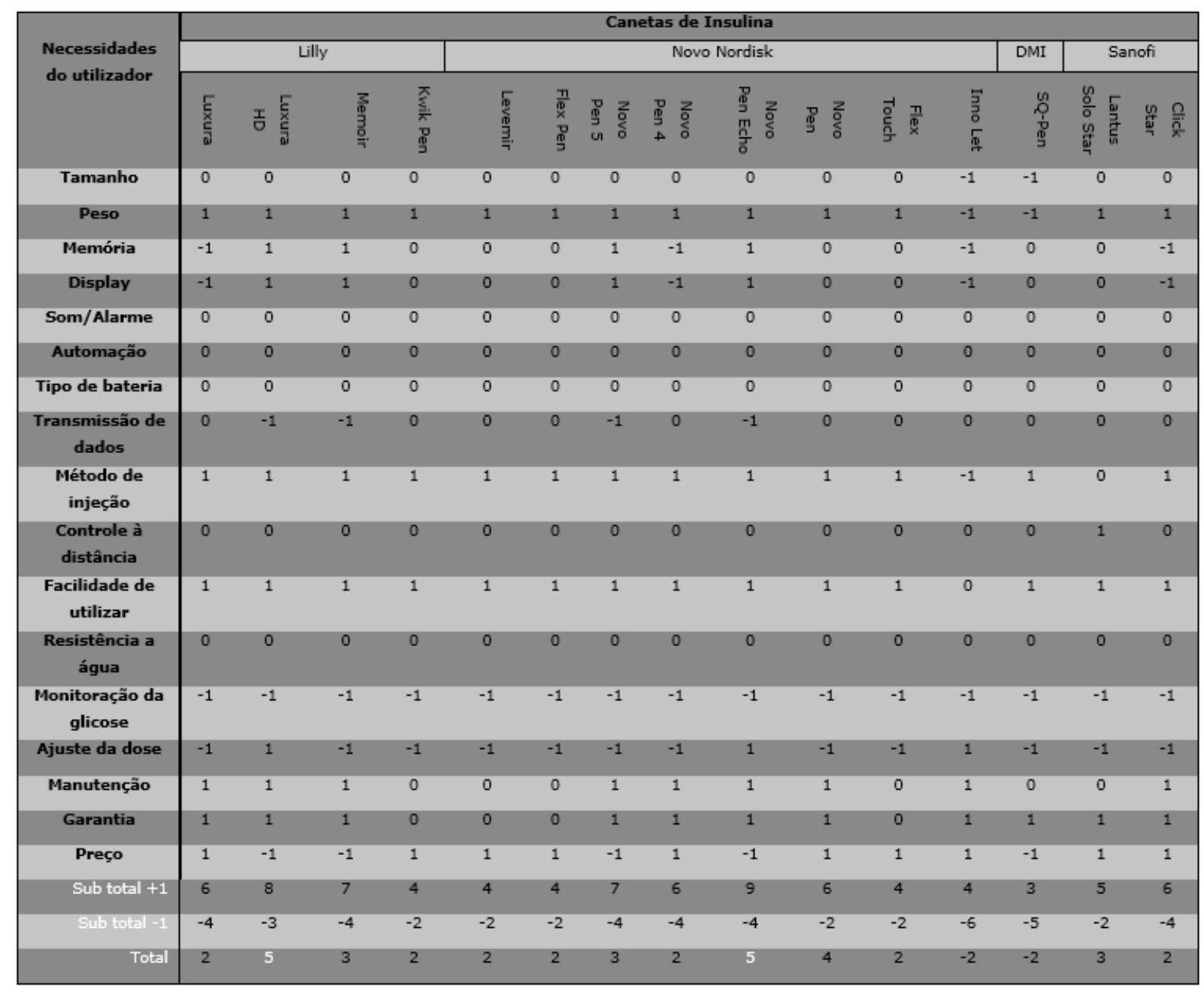

Tabela 2: Tabela comparativa das canetas existentes no mercado

Nesta análise identificou duas canetas que se encaixam com maior pontuação nas necessidades do utilizador: a caneta reutilizável Luxura HD (Lilly) e a caneta reutilizável NovoPen Echo (Novo Nordisk), estas não são as mais utilizadas entre os diabéticos inquiridos por serem recentemente comercializadas. A principal diferença destas às outras canetas é o display com função memória e o ajuste das doses em 0.5 $\mathrm{UI}$, essencial na administração de insulina em crianças diabéticas.

Nesta outra análise, a bomba de infusão Accu-Chek Combo (Roche) se destaca dos outros dispositivos, sendo o dispositivo que se encaixa em quase todas as necessidades do utilizador. O principal ponto negativo neste tipo de dispositivo é o preço e o custo de manutenção, principalmente quando comparados com as canetas de insulina, nem sempre sendo acessivel a todos.

$\mathrm{Na}$ Tabela 4 podem-se perceber as vantagens e desvantagens destes dispositivos.

Por um lado, há dispositivos que permitem maior liberdade do diabético porém, as canetas necessitam de múltiplas injeções durante o dia e maior discrição na administração. Por outro, tem-se um dispositivo fixo no corpo e de grandes dimensões, bombas de infusão, trazendo mais qualidade de vida ao utilizador, mas mais limitações nas atividades diárias. Apesar das diversas vantagens e desvantagens e a eficácia no tratamento do diabetes através destes dispositivos, existe uma lacuna entre estes dois métodos de tratamento, ou seja, não existindo nenhum dispositivo dedicado 


\begin{tabular}{|c|c|c|c|c|c|}
\hline \multirow{3}{*}{$\begin{array}{l}\text { Especificações do } \\
\text { produto } \\
\text { Necessidades do } \\
\text { utilizador }\end{array}$} & \multicolumn{5}{|c|}{ Bombas de infusão } \\
\hline & \multicolumn{2}{|c|}{ Medtronic } & \multicolumn{3}{|c|}{ Roche } \\
\hline & Paradigm & $\begin{array}{c}\text { Paradigm } \\
\text { Veo }\end{array}$ & $\begin{array}{l}\text { Accu-Chek } \\
\text { Combo }\end{array}$ & $\begin{array}{l}\text { Accu-Chek } \\
\text { Spirit }\end{array}$ & $\begin{array}{l}\text { Accu-Chek D- } \\
\text { TRONplus }\end{array}$ \\
\hline Tamanho & -1 & -1 & -1 & -1 & -1 \\
\hline Peso & -1 & -1 & -1 & -1 & -1 \\
\hline Memória & 1 & 1 & 1 & 1 & 1 \\
\hline Display & 1 & 1 & 1 & 1 & 1 \\
\hline Som/Alarme & 1 & 1 & 1 & 1 & 1 \\
\hline Automação & 0 & 1 & 1 & 0 & 0 \\
\hline Tipo de bateria & -1 & -1 & -1 & -1 & -1 \\
\hline $\begin{array}{c}\text { Transmissão de } \\
\text { dados }\end{array}$ & 0 & 0 & 1 & 1 & 0 \\
\hline Método de injeção & 1 & 1 & 1 & 1 & 1 \\
\hline Controle à distância & 0 & 1 & 1 & 0 & 0 \\
\hline Facilidade de utilizar & 0 & 0 & 0 & 0 & 0 \\
\hline Resistência a água & 1 & 1 & 1 & 1 & 1 \\
\hline $\begin{array}{c}\text { Monitoração da } \\
\text { glicose }\end{array}$ & -1 & 1 & 1 & -1 & -1 \\
\hline Ajuste da dose & 1 & 1 & 1 & 1 & 1 \\
\hline Manutenção & -1 & -1 & -1 & -1 & -1 \\
\hline Garantia & 1 & 1 & 1 & 1 & 1 \\
\hline Preço & 0 & -1 & -1 & -1 & -1 \\
\hline Sub total +1 & 7 & 10 & 11 & 8 & 7 \\
\hline Sub total -1 & -5 & -5 & -5 & -6 & -6 \\
\hline Total & 2 & 5 & 6 & 2 & 1 \\
\hline
\end{tabular}

\section{Identificação das necessidades}

Com base na recolha acima, identificam-se alguns fatores que interferem na realização da administração de insulina. Para identificar as necessidades do consumidor foi construída a Tabela 5. Nesta tabela, o ranking das necessidades identifica os requisitos, por relevância, tendo em conta as necessidades do utilizador, identificados na pesquisa realizada através da questão "como gostaria que o dispositivo de administração de insulina fosse?".

Por meio do ranking das necessidades construído percebe-se que os utilizadores preferem, sobretudo, um dispositivo mais discreto e um dispositivo autônomo; isto é, sem a necessidade de realizar picadas para verificar os níveis de glicose e posteriormente administrar a insulina, tendo um dispositivo que fizesse estas duas tarefas simultaneamente e automaticamente. Em outro ponto, é abordada a necessidade da redução do tamanho dos dispositivos, facilitando seu transporte e armazenamento. Outro aspecto é a necessidade do dispositivo ser mais intuitivo, tendo uma rápida compreensão de sua utilização e praticidade. Alguns dos utilizadores de caneta (17\%) prefeririam que seu dispositivo fosse ou uma bomba de infusão ou algo que se apro- 


\begin{tabular}{|c|c|c|}
\hline & Vantagens & Desvantagens \\
\hline 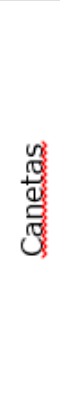 & 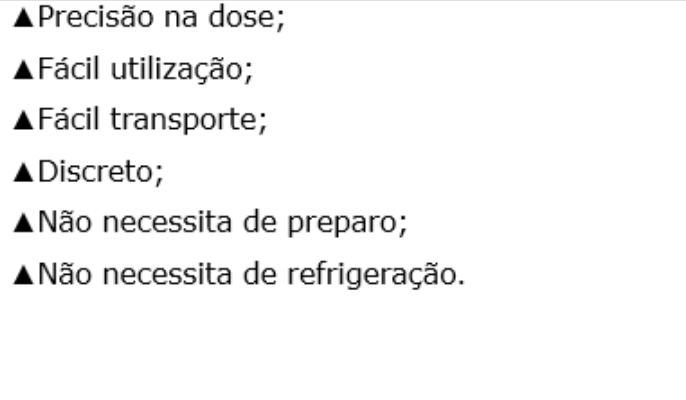 & 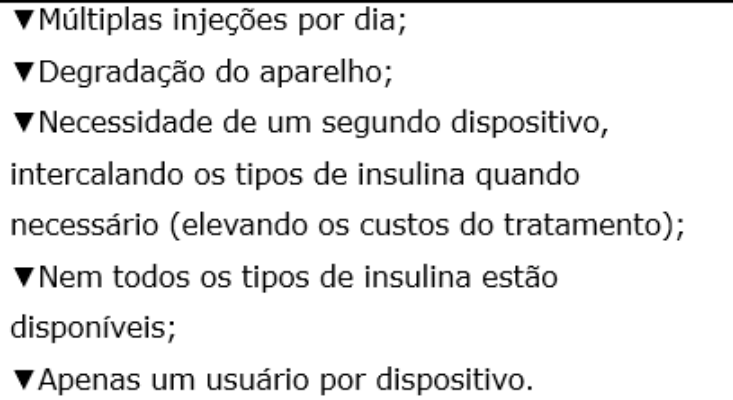 \\
\hline 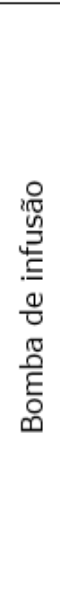 & 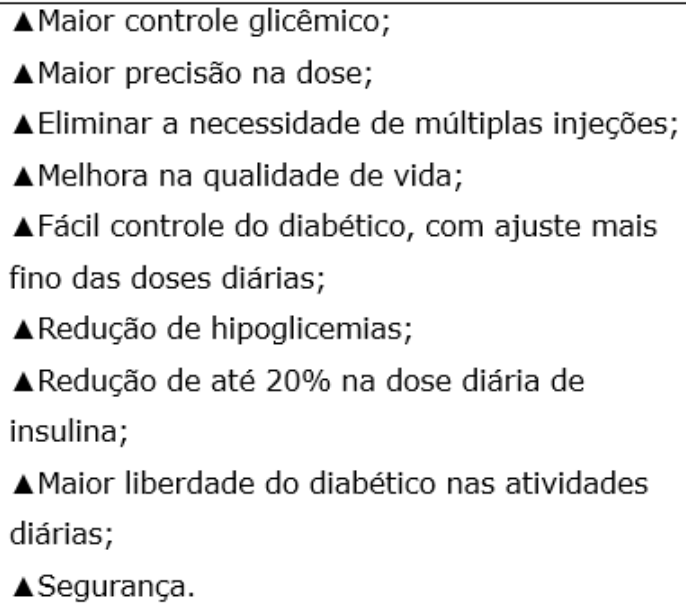 & 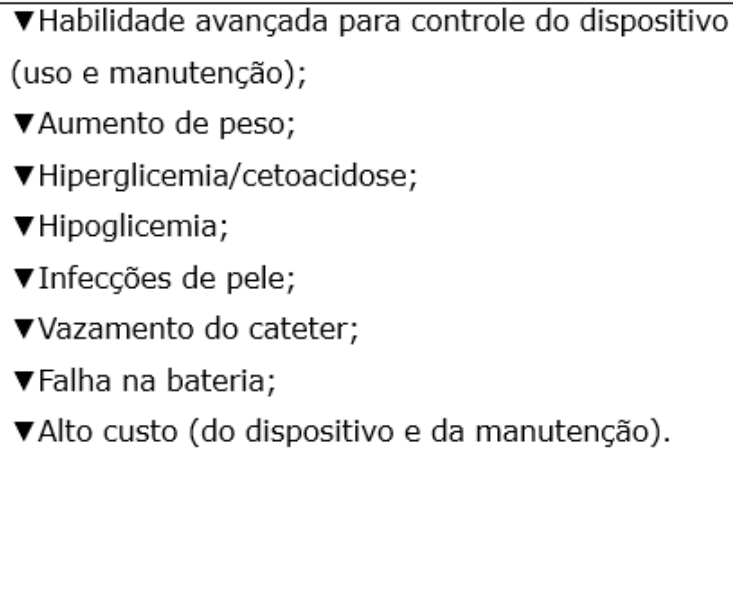 \\
\hline
\end{tabular}

ximasse de uma bomba de infusão, ajudando também na redução de picadas diárias.

\begin{tabular}{|l|c|}
\hline \multicolumn{1}{|c|}{ Necessidade } & $\begin{array}{c}\text { Citações pelo } \\
\text { utilizador } \\
\text { (Total de } \\
107 \text { ) }\end{array}$ \\
\hline Dispositivo mais discreto & 18 \\
\hline $\begin{array}{l}\text { Dispositivo autônomo, realizasse a medição e administração sem } \\
\text { intervenção do diabético }\end{array}$ & 17 \\
\hline Dispositivo menor & 14 \\
\hline Mais intuitivo (prático) & 12 \\
\hline Indolor & 9 \\
\hline Não injetável (intranasal ou comprimido) & 7 \\
\hline Que fosse como uma bomba de infusão & 6 \\
\hline Dispositivo no interior do corpo (autônomo) & 5 \\
\hline Sem a presença de agulha & 4 \\
\hline Aspecto apelativo & 3 \\
\hline Bomba de infusão sem fio & 3 \\
\hline Necessário menos picadas & 3 \\
\hline Alertasse o nível de glicemia & 2 \\
\hline Controle por comando & 2 \\
\hline Desse para usar em qualquer parte do corpo & 1 \\
\hline Fixo no corpo & 1 \\
\hline
\end{tabular}

Tabela 4: Vantagens e desvantagens das canetas e bombas (adaptado de MAIA, 2002 [9] e MINICUCCI, 2008 [10])

Tabela 5: Ranking das necessidades dos utilizadores

Após a recolha das necessidades dos utilizadores, foi constituída uma árvore hierárquica das necessidades, dividida em três níveis separados conforme a catego- 


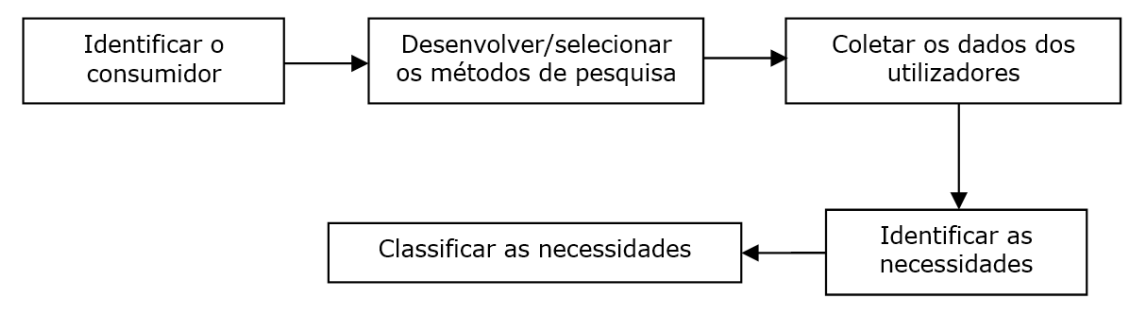

ria, Figura 4, ajudando a perceber em que categoria cada necessidade se encaixa [11].

Figura 4: Árvore das necessidades [12]

Por meio da árvore das necessidades construída fez-se a divisão das necessidades em cada categoria do dispositivo. Para mais, com base na mesma árvore, foi possível estabelecer os pré-requisitos identificados entre os utilizadores, essenciais para iniciar o projeto de desenvolvimento de um novo dispositivo adequado para crianças:

- Administração de insulina;

- Dispositivo sem fio;

- Redução do número de picadas;

- Não exceder as dimensões dos dispositivos atuais;

- Ser mais leve, facilitando o transporte;

- Precisão, utilizando valores mínimos de 0.5 UI para administração [4]. 


\section{CONCLUSÃO}

O projeto foi dividido em três grandes fases, a recolha de informações acerca da doença, identificação dos dispositivos mais utilizados e das novas tecnologias para o tratamento da diabetes e a pesquisa com utilizador. O conhecimento da doença enfatizou como esta vem sendo abordada desde seus primórdios, como funcionam os tratamentos para redução das complicações, quais os métodos utilizados para este tratamento e como a criança diabética se sente diante destas exigências diárias. Esta fase foi fundamental para se inserir no ambiente em que essas crianças vivem, entendendo suas necessidades diárias.

Em seguida na fase de identificação das necessidades, o contato direto com os doentes possibilitou mais-valia para identificação das suas limitações e a vivência durante o dia entre o diabético e seu tratamento. A aplicação dos inquéritos online ajudou a fortalecer alguns critérios já identificados durante a primeira pesquisa. Num todo, esta análise permitiu identificar claramente a ausência de um dispositivo menor e mais discreto para administração de insulina.

O design mais que tudo está diretamente ligado ao desenvolvimento de produtos, ainda mais quando se trata de dispositivos médicos devido à importância que eles têm na vida diária do seu utilizador. Apesar de já existirem alguns dispositivos médicos relativamente eficientes no tratamento da diabetes, crianças e adolescentes apresentam uma necessidade quanto a um dispositivo novo que atenda suas necessidades.

A pesquisa realizada apresenta diversos critérios que devem ser levados em consideração durante o desenvolvimento de um dispositivo para este público. Demonstrando que há oportunidade de criação de um dispositivo médico para controle desta doença em crianças e adolescentes. Portanto, esta é uma tarefa para designers aplicarem seus conhecimentos e desenvolverem um produto que supra as necessidades aqui apresentadas.

\section{REFERÊNCIAS}

[1] NUNES, José Silva. Diabetes: uma abordagem global. Lisboa: Sociedade Portuguesa de Endocrinologia, Diabetes e Metabolismo, 2010.

[2] MINANNI, Carlos André et. al. Abordagem integral do adolescente com diabetes. Adolescência e Saúde. 2010;7(1): 45-52.

[3] CASTRO, Ampario del Rocio Vintimilla; GROSSI, Sonia Aurora Alves. Reutilização de seringas descartáveis no domicílio de crianças e adolescentes com diabetes mellitus. Revista da Escola de Enfermagem da USP. São Paulo, v. 41, n. 2, June, 2007. Disponível em: $\quad<$ http://www.scielo.br/scielo.php?script=sci_arttext\&pid=S0080-62342007000200003\&lng=en\&nrm=iso $>$. Acesso em 09 Set. 2013.

[4] GROSSI, Sonia Aurora Alves; PASCALI, Paula Maria. Cuidados de enfermagem em 
diabetes mellitus. Manual de Enfermagem. São Paulo: Sociedade Brasileira de Diabetes, 2009.

[5] DUARTE, Rui. Diabetologia Clínica. 3ạ Edição. Lisboa: Lidel, 2002.

[6] PIRES, Antonio Carlos; CHACRA, Antonio Roberto. A evolução da insulinoterapia no diabetes melito tipo 1. Arquivos Brasileiros de Endocrinologia e Metabologia, São Paulo, v.52, n.2, Mar. 2008. Disponível em <http://www.scielo.br/scielo.php?script=sci_arttext\&pid=S0004-27302008000200014\&lng=en\&nrm=iso $>$. Acesso em 30 Jan. 2013.

[7] OLIVEIRA, Maria Camargo de. Preparo e aplicação de insulina sem mistério. São Paulo: Centro BD de Educação em Diabetes, 2011.

[8] SANTOS, Isa C. T. Product development methodologies: the case of medical devices. 2012. Thesis submitted to the Faculdade de Engenharia da Universidade do Porto for the degree of Doctor of Philosophy in Leaders for Technical Industries of the MIT - Portugal Program.

[9] MAIA, Frederico F. Ribeiro; ARAUJO, Levimar R.. Uso da caneta injetora de insulina no tratamento do diabetes mellitus tipo 1. Jornal de Pediatria. (Rio J.), Porto Alegre, v. 78, n. 3, 2002. Disponível em: <http://www.scielo.br/scielo.php?script=sci_arttex$\underline{t \& p i d=S 0021-75572002000300004 \& \operatorname{lng}=e n \& n r m=i s o}>$. Acesso em: 31 Jan. 2013.

[10] MINICUCCI, Walter José. Uso de bomba de infusão subcutânea de insulina e suas indicações. Arquivos Brasileiros de Endocrinologia e Metabologia. São Paulo, v.52, n.2, Mar. 2008.

[11] SHUM, Paul; LIN, Grier. A world class new product development best pratices model. International Journal of Production Researche. Australia, v.45, n.7, 2007.

[12] TESCKE, Lucas R. Desenvolvimento de um dispositivo de administração de insulina para crianças diabéticas. 2013. 150 f. Dissertação (Mestrado) - Faculdade de Engenharia da Universidade do Porto, Curso de Mestrado em Design Industrial. 


\section{APÊNDICE A QUESTIONÁRIO DIABÉTICOS}

No âmbito do Mestrado em Design Industrial da Faculdade de Engenharia da Universidade do Porto, estou a desenvolver um novo dispositivo para a administração de insulina em crianças e adolescentes. Este inquérito é anónimo e tem como objetivo identificar as características que o novo produto deverá ter. A qualquer momento pode desistir de responder a este inquérito. Desde já agradecemos a sua colaboração e poderá esclarecer qualquer dúvida através dos contatos: Lucas Renato Tescke - lucastescke@gmail.com, Isa Santos - isa.santos@fe.up.pt ou Prof. João Manuel Tavares - tavares@,fe.up.pt.

1.Sexo

( ) Masculino

( ) Feminino

2.Idade?

3.Há quanto tempo foi diagnosticada a diabetes?

( ) 1 ano

() 2 anos

( ) 3 ou mais

4.Que tipo de insulina (ação) administra?
( ) Lenta
( ) Intermédia
( ) Rápida
( ) Ultrarrápida

5.Quem é o responsável pela aplicação da insulina?

( ) Eu

( ) Os meus pais/avós

( ) Outro. Qual?

6.Que dispositivo utiliza para administrar a insulina?

( ) Seringa de Insulina

( ) Caneta de Insulina

( ) Caneta de Insulina Descartável

( ) Bomba de Infusão de Insulina

7.Quantas injeções de insulina administra diariamente? 

( ) 1
( ) 2
( ) 3
( ) 4 ou mais

8.Durante o dia, quando administra a insulina?

( ) Antes do pequeno-almoço

( ) Depois do pequeno-almoço

( ) Antes do almoço

( ) Depois do almoço

( ) Antes do jantar

( ) Depois do jantar

( ) Antes de dormir

9. Onde costuma fazer a administração das injeções de insulina?
( ) Casa
( ) Escola
( ) Outro:

10.Em que zona do corpo costuma realizar a aplicação de insulina?
( ) Braço
( ) Barriga
( ) Coxas
( ) Nádegas
( ) Outro. Qual?

11.Quais são as dificuldades para administrar a insulina?
( ) Medo
( ) Insegurança
( ) Não vejo
( ) Não percebo como funciona
( ) $\bigcirc$ médico não deixa
( ) Os pais não deixam

12. Quem escolheu o dispositivo para administrar a insulina?
( ) Eu
( ) Médico
( ) Os pais

13. Quais os fatores que contribuíram para a escolha do dispositivo para administrar insulina?

( ) É fácil de utilizar

() Segurança

( ) Aspecto apelativo

( ) Aspecto formal 
( ) Discreto, passa despercebido

( )Outro. Qual?

14. Qual a marca e modelo do dispositivo que utiliza para administrar insulina? ()

15.Realiza a administração conforme as orientações médicas?

() $\operatorname{Sim}$

()

Caso não realiza, por quê?

() Medo

() Dor

( ) Não quer

() Insegurança

() Vergonha

() Esqueço-me

() Outro:

16.Como se sente ao dispositivo utilizado atualmente?

( ) Muito satisfeito

() Satisfeito

() Neutro

() Insatisfeito

() Muito insatisfeito

17.Que atividades gosta de fazer?

() Ler

() Dormir

() Correr

() Brincar com amigos

() Nadar

( ) Ver televisão

( ) Jogar vôlei

( ) Jogar futebol

( ) Jogar computador/consola

() Jogar Basquetebol

() Navegar na Internet

() Estudar

( ) Prefere não fazer nada

18.Como gostaria que o dispositivo de administração de insulina fosse? 
19.Gostaria que o dispositivo de administração fosse discreto, passasse despercebido pelos amigos?

( ) Sim

( ) Não

20.Gostaria que o dispositivo emitisse um sinal sonoro após injetar a insulina?

( ) Sim

( ) Não

21.Gostaria que vocês mesmo pudesse realizar a manutenção do dispositivo (troca de insulina, regulagem da quantidade), sem ajuda dos pais ou um profissional da saúde?

( ) Sou utilizador independente

( ) Sim

( ) Não

22.Gostaria que o dispositivo tivesse cor?

( ) Sim

( ) Não

Obrigado pela sua colaboração.

Anotações durante a administração:

O que está a fazer? Sente-se bem? Tens alguma dificuldade? O que achas que poderia melhorar? 